\title{
Conscientização em alusão ao Outubro Rosa: vamos falar sobre câncer de mama?
}

\author{
Awareness in allusion to Pink October: let's talk about breast câncer? \\ Conciencia em alusión al Octubre Rosa: hablemos de câncer de mama?
}

Ana Karina Rodrigues Coelho ORCID: https://orcid.org/0000-0001-8504-689X

Centro Universitário Fibra, Brasil E-mail: anna.karinna1999@gmail.com

João de Souza Mendonça ORCID: https://orcid.org/0000-0002-2204-5010

Universidade da Amazônia, Brasil

E-mail: joaosm.farmaco@gmail.com

Amanda Almeida Vieira

ORCID: https://orcid.org/0000-0003-2211-0115

Centro Universitário do Estado Pará, Brasil

E-mail: amandaav2000@gmail.com

Barbara Maria Neves Mendonça Luz

ORCID: https://orcid.org/0000-0001-7907-9337

Centro Universitário do Estado do Pará, Brasil

E-mail: barbaramendoncaluz@outlook.com

Marcello Vieira dos Santos

ORCID: https://orcid.org/0000-0002-3356-1619

Centro Universitário do Estado do Pará, Brasil

E-mail: marcellosantos277@gmail.com

Matheus Osório Lima

ORCID: https://orcid.org/0000-0001-9467-2972

Centro Universitário do Estado do Pará, Brasil

E-mail: osoriomatheus@hotmail.com

Camila Ribeiro Cardoso

ORCID: https://orcid.org/0000-0002-3612-0341

Centro Universitário Fibra, Brasil

E-mail: enfa.camilasantos@gmail.com

Manuele Figueira Printes

ORCID: https://orcid.org/0000-0003-3286-0497

Universidade Norte do Paraná, Brasil

E-mail: figueiramanuele9@gmail.com

Ana Paula Cecilia Braga

ORCID: https://orcid.org/0000-0002-1414-1061

Centro Universitário do Estado do Pará, Brasil

E-mail: anapaulaceciliabraga@gmail.com

Ana Carolina da Silva Borges

ORCID: https://orcid.org/0000-0003-2454-331X

Escola Superior da Amazônia, Brasil

E-mail: a.sborges20@gmail.com

Leidiane de Jesus da Costa Santos

ORCID: https://orcid.org/0000-0002-9208-2319

Escola Superior da Amazônia, Brasil

E-mail: leidianesantos2023@gmail.com

Tâmia Rayara Carvalho Araújo da Silva

ORCID: https://orcid.org/0000-0003-4713-7314

Universidade da Amazônia, Brasil

E-mail: ta.mia10@hotmail.com

Daniela Vergilio Grassi

ORCID: https://orcid.org/0000-0001-7208-9284

Centro Universitário do Estado do Pará, Brasil

E-mail:danielavergilio06@gmail.com

Lorena Barros Bahia

ORCID: https://orcid.org/0000-0003-4444-3850

Centro Universitário do Estado do Pará, Brasil

E-mail:lorenabbahia@gmail.com

Tamires Costa Franco

ORCID: https://orcid.org/0000-0002-8978-4176

Universidade da Amazônia, Brasil

E-mail: tamiresfranco98@gmail.com 


\begin{abstract}
Resumo
O câncer de mama ocorre quando há uma proliferação exacerbada das células, ou seja, uma multiplicação rápida e desordenada das células que pode ocorrer por fatores ambientais e genéticos. $\mathrm{O}$ câncer de mama destaca-se como o segundo tipo de neoplasia mais frequente mundialmente, apenas atrás do câncer de pele não melanoma, que ocupa o primeiro lugar. Os fatores de risco que estão mais vinculados ao desenvolvimento do câncer de mama são a idade avançada, características reprodutivas, histórico familiar de câncer e hábitos de vida. Trata-se de um estudo de caráter descritivo, do tipo relato de experiência, realizado em uma Unidade Básica de Saúde, em Belém, estado do Pará, durante uma ação realizada na referida unidade sobre a conscientização na prevenção e tratamento do câncer de mama. As mulheres que estavam participando da roda de conversa, ouviram atentamente os relatos e explicações afim de realizarem o " toque" nas mamas como forma de detecção precoce, com a intenção de procurarem um serviço médico ao notarem algo anormal. A assistência multiprofissional é crucial para que haja uma incidência menor nas taxas de mortalidade causadas pelo câncer de mama. Dessa maneira, há necessidades de capacitação de todos os profissionais na atenção básica, proporcionarem ações educativas que esclareçam sobre as formas de prevenção e tratamento do câncer mamário.
\end{abstract}

Palavras-chave: Autoexame; Câncer; Educação em saúde.

\begin{abstract}
Breast cancer occurs when there is an exacerbated proliferation of cells, that is, a rapid and disordered multiplication of cells that can occur due to environmental and genetic factors. Breast cancer stands out as the second most frequent type of neoplasm worldwide, just behind non-melanoma skin cancer, which occupies the first place. The risk factors that are most linked to the development of breast cancer are advanced age, reproductive characteristics, family history of cancer and lifestyle habits. This is a descriptive study, of the experience report type, carried out in a Basic Health Unit, in Belém, Pará, during an action carried out in that unit on raising awareness in the prevention and treatment of breast cancer. The women who were participating in the conversation circle listened carefully to the reports and explanations in order to perform the 'touch' on the breasts as a form of early detection, with the intention of seeking medical care when they noticed something abnormal. Multiprofessional care is crucial so that there is a lower incidence of mortality rates caused by breast cancer. Thus, there is a need for training of all professionals in primary care, providing educational actions that clarify the forms of prevention and treatment of breast cancer.
\end{abstract}

Keywords: Self exam; Cancer; Health education.

\title{
Resumen
}

El cáncer de mama ocurre cuando hay una proliferación celular exacerbada, es decir, una multiplicación celular rápida y desordenada que puede ocurrir debido a factores ambientales y genéticos. El cáncer de mama se destaca como el segundo tipo de neoplasia más frecuente en todo el mundo, solo por detrás del cáncer de piel no melanoma, que ocupa el primer lugar. Los factores de riesgo más relacionados con el desarrollo del cáncer de mama son la edad avanzada, las características reproductivas, los antecedentes familiares de cáncer y los hábitos de vida. Se trata de un estudio descriptivo, del tipo relato de experiencia, realizado en una Unidad Básica de Salud, en Belém, Pará, durante una acción realizada en dicha unidad de sensibilización en la prevención y tratamiento del cáncer de mama. Las mujeres que participaban en el círculo de conversación escuchaban atentamente los relatos y explicaciones para realizar el 'toque' en las mamas como forma de detección precoz, con la intención de buscar atención médica cuando notaran algo anormal. La atención multiprofesional es fundamental para que haya una menor incidencia de tasas de mortalidad por cáncer de mama. Por tanto, existe la necesidad de formar a todos los profesionales de la atención primaria, proporcionando acciones educativas que esclarezcan las formas de prevención y tratamiento del cáncer de mama.

Palabras clave: Autoexamen; Cáncer; Educación para la salud.

\section{Introdução}

No século XX ocorreram transformações radicais nas formas de compreender, apresentar e abordar sobre o câncer. (Teixeira \& Neto, 2020).

O câncer de mama é um tumor maligno mais incidente em mulheres sendo um problema de saúde pública em virtude do seu diagnóstico tardio e por sua alta taxa de mortalidade (Leite et al., 2021).

Segundo o Instituto Nacional do Câncer (INCA, 2021), o câncer de mama é uma doença causada pela multiplicação desordenada das células anormais da mama, que forma um tumor com potencial de invadir outros órgãos. Há vários tipos de câncer de mama, alguns com desenvolvimento mais rápidos, e outros com desenvolvimento mais lento. Quando tratados adequadamente, evolui para um bom prognóstico. 
De acordo com dados publicados pelo INCA (2020), a taxa de mortalidade por câncer de mama é a primeira causa de morte por câncer da população brasileira feminina, com 14,23 óbitos para 100.000 mulheres. Sendo necessário destacar a incidência do câncer de mama, os dados tendem a crescer de forma progressiva a partir dos 40 anos, assim como a mortalidade por essa neoplasia.

O câncer de mama em suas características anormais tem como aspectos de anormalidades proliferações nos lóbulos e ductos mamários que inclui carcinoma invasivo, hiperplasia, hiperplasia atípica e carcinoma em situ, sendo um edema cutâneo similar a casca de laranja, podendo aparecer linfonodos palpáveis na axila (Bravo et al.,2021).

O controle dessa doença se dá através da detecção precoce, na qual a lesão se restringe ao parênquima mamário, com um tamanho de no máximo três centímetros, permitindo o uso de recursos terapêuticos menos mutiladores e maior possibilidade de cura (Silva \& Riul, 2015).

A partir dos anos de 1940, a criação do Serviço Nacional do Câncer, da Sociedade Brasileira de Cancerologia, bem como instituições de assistências aos cancerosos pelo país, reconfigurou o campo de discussões sobre o câncer de mama. A ampliação da utilização de mastectomia, a introdução de novas tecnologias para diagnóstico e a introdução de campo cirúrgico mais direcionado à doença, levou a novos debates sobre o combate aos tumores no seio (Teixeira \& Neto, 2020).

Entretanto, a relação de fatores de risco é fundamental para o desenvolvimento do câncer de mama, ressaltando o histórico familiar de câncer de mama, câncer de ovário e outros cânceres associados a mutações de linha germinativa, como por exemplo o câncer de próstata, que tem associação direta com o risco aumentado para o câncer de mama (Silva et al.,2021)

Segundo Silva (2021), fatores hormonais como o uso de anticoncepcional oral e terapia de reposição hormonal durante a menopausa, além de má alimentação, obesidade, sedentarismo, configuram-se como fatores de risco para um câncer de mama.

No contexto de Estratégia de Saúde da Família (ESF), as ações para o controle do câncer de mama são direcionadas para a promoção da saúde e prevenção de doenças, além do diagnóstico precoce e apoio ao tratamento instituído na atenção secundária e terciária (Silva et al.,2021).

Os métodos de diagnóstico da doença são a mamografia e o exame clínico, além de outros como ultrassonografia, ressonância, exames de sangue, raio-X, cintilografia, biópsia, exames citopatológico e histopatológico e exames de BRCA1 e BRCA2. Porém, mesmo com todos esses métodos de diagnóstico, o principal desafio ainda está em se ter um diagnóstico precoce da doença, algo que só seria resolvido com um maior investimento na saúde pública, visando uma maior acessibilidade aos métodos de prevenção e maior informação das mulheres sobre o câncer de mama, pois a maioria dos casos é diagnosticado tardiamente dificultando as chances de sobrevida das pacientes (Bernardes et al., 2020).

O câncer de mama deve ser abordado por uma equipe multidisciplinar visando o tratamento integral da paciente. As modalidades terapêuticas disponíveis atualmente são as cirúrgicas e as radioterápicas para o tratamento locorregional, e a hormonioterapia e a quimioterapia para o tratamento sistêmico (Silva \& Riul, 2015).

O objetivo deste estudo é descrever o desempenho de acadêmicos de diferentes cursos utilizando a educação em saúde como estratégia de conscientização para a detecção precoce ao câncer de mama.

\section{Metodologia}

Este estudo caracteriza-se como descritivo, do tipo relato de experiência, realizado em uma Unidade Básica de Saúde, no Estado do Pará, no mês de outubro de 2021.

Durante o mês de outubro ocorrem em diversos locais campanhas de conscientização em relação a prevenção e tratamento do câncer de mama, marcado pelo Outubro Rosa. 
No referido posto de saúde, ocorreu a conscientização que se deu com ajuda de profissionais que já atuam na área, bem como acadêmicos dos cursos de enfermagem, nutrição, biomedicina, fisioterapia, medicina e psicologia. A fim de explicar e demonstrar como a mulher pode se auto avaliar em casa para detectar possíveis alterações na mama. Além de enfatizar os fatores de riscos que podem ser predisponentes a esse tipo de neoplasia.

É fundamental a ação anualmente de campanhas que possam promover a detecção precoce do câncer de mama, com base nisso foi montado uma roda de conversa para elucidar a importância da mulher reconhecer seu próprio corpo, e as formas de tratamento se já houver diagnóstico para o câncer de mama.

Foi empregado uma linguagem acessível, onde foi explicado: o que é câncer de mama; quais são os fatores de risco; sinais e sintomas; como é feito o diagnóstico; suas formas de tratamento e medidas de prevenção.

Foi utilizado folder explicativo de nutrição para uma alimentação adequada, sem o consumo de enlatados e " fast food", e o uso da " mamamiga" que é um demonstrativo da mama dividido em quatro quadrantes, onde é possível realizar o toque e identificar os achados de uma mama normal e de uma mama com nódulos.

O autoexame de mama é utilizado como uma forma de prevenção e diagnóstico precoce, caracteriza-se como um processo simples e indolor que auxilia na detecção do câncer em seu estágio inicial que pode inclusive aparecer na forma de pequenos nódulos mamários. A realização correta do autoexame ocorre uma vez ao mês, durante a segunda semana após a menstruação. A descoberta de nódulos na mama propicia procura de um especialista no intuito de evitar maiores danos, facilitar a terapêutica e possivelmente, a cura (Santana et al., 2021).

O fundamento teórico decorreu pela busca bibliográfica em bases de dados científicas digitais, sendo elas: Scientific Electronic Library Online (SCIELO) e Biblioteca Virtual em Saúde (BVS), com a utilização de palavras-chaves colhidas nos

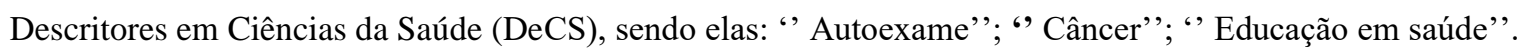

\section{Resultados}

Durante a explicação, que contou com cerca de 20 mulheres de diversas faixas etárias, foi possível perceber que todas estavam atentas no assunto que foi abordado.

A importância de debatermos este assunto na atenção primária é importante para uma detecção precoce, visto que algumas mulheres podem sentir algum desconforto há anos e podem ignorar por falta de informação ou auxílio médico inadequado.

A equipe multiprofissional tanto para detecção quanto para o tratamento do câncer de mama é fundamental na vida do paciente, pois, além de ser uma questão de estética na maioria das vezes, há um abalo mental e social na vida daquela mulher, independente do grau no qual o câncer se encontra.

Foi abordado o enfrentamento desde o diagnóstico de câncer de mama, até uma possível cirurgia, além de destacar quando confirmado o tratamento quimioterápico, que na sua alta incidência afeta esteticamente e psicologicamente a vida da mulher. Com isso, a equipe multiprofissional presente pode enfatizar cada eixo para que a mulher tenha a autonomia de reconhecer seu próprio corpo.

Segundo o INCA (2021) o objetivo da atenção primária é impedir que o câncer se desenvolva, isso inclui evitar a exposição a fatores de risco e a adoção de uma vida saudável.

Logo, elucidado a questão de fatores de risco que podem prejudicar ainda mais a vida da mulher, o enfoque na boa alimentação, na prática de exercícios físicos, foram os eixos mais abordados para que se tenha uma vida de qualidade e assim diminuir a incidência de casos, evitando o uso de bebidas alcoólicas, evitar comer carne processada, manter um bom peso corporal de acordo com a idade, realizar exame preventivo, entre outros fatores. 
Diante a pesquisa teórica e prática, podemos notar a falta de comunicação na atenção primária em relação à prevenção, diagnóstico e tratamento relacionado ao câncer de mamário.

Durante a abordagem, foi debatido os sinais e sintomas, e uma breve ilustração contando como a "mamamiga", onde é possível realizar o toque em achados mamários normais e alterados, contando com esse suporte às mulheres presentes foram estimulada a realizar esse exame físico na sua própria residência, contendo dicas e informações de como é realizado.

Ademais foi abordado a questão do diagnóstico e consultas periódicas para exames de rotina que na maioria das vezes podem apontar algo anormal das mamas, a importância do acompanhamento com médicos e enfermeiros, podendo sempre tirar as dúvidas para que fiquem bem esclarecidas quanto a um possível diagnóstico de câncer de mama.

Vale ressaltar que o Sistema Único de Saúde (SUS) oferece tratamento gratuito para o câncer de mama, e na maioria das vezes muitas mulheres não recebem o apoio familiar para buscar o tratamento correto e adequado, acarretando na evolução da doença.

Em perspectiva do assunto abordado, vale salientar o ponto de vista para o desempenho acadêmico, o qual nos favorece contato mais próximo com o paciente, a fim de elucidar esse tema de uma maneira recreativa, com a finalidade de repassar nossos conhecimentos de maneira clara. De maneira, que enfatiza o tema, aperfeiçoa nossos conhecimentos, e proporciona de certa forma uma " chamada" na comunidade, afim de obter mais engajamento e conscientização nas classes mais vulneráveis.

Sendo assim, foi possível repassar o propósito que foi conscientizar essas mulheres em uma linguagem simples e de fácil entendimento, para que possamos futuramente reduzir os casos de incidência causados pelo câncer de mama. Visto que, quanto mais precoce a detecção, melhor serão os resultados no tratamento, por isso é importante desmistificar a doença como uma " decisão" de morte.

\section{Discussão}

No Brasil, de acordo com o Instituto Nacional de Câncer, estima-se o aparecimento de 66.280 casos novos casos em 2020. A evolução da doença pode variar de acordo com o tipo de tumor, sendo mais tardia ou precoce, podendo ou não invadir outros tecidos (Lourenço et al., 2020).

Dados os vários meios de diagnóstico e a contínua falta de detecção precoce a partir do rastreamento da neoplasia é significativo abordar os meios de diagnóstico do câncer de mama e sua eficiência para a redução da mortalidade, além de assegurar uma melhoria na qualidade da saúde das mulheres com uma maior divulgação e conscientização sobre o assunto. (Bernardes et al., 2020).

A Educação em Saúde é uma estratégia que potencializa o cuidado interdisciplinar ao envolver atividades educativas na assistência ao paciente, utilizando recursos disponíveis nos serviços de saúde, sejam públicos ou privados. Sendo, estas ações importantes para a promoção da qualidade de vida e para o desenvolvimento de tarefas diárias das pessoas (Vieira et al., 2017).

Baseado na experiência vivida de promover uma roda de conversa em relação a importância da prevenção do câncer de mama, foi possível repassar passo a passo para detecção, visto que em algumas situações essas mulheres não tem liberdade de falarem o que pensam por medo de serem julgados e por acharem que o câncer é o fim de tudo.

$\mathrm{Na}$ atual circunstância, o modelo de ensino tradicional vem sendo gradativamente substituído por novas tendências e implicações as quais apontam para a necessidade da formação de um profissional crítico-reflexivo, capaz de transverter a realidade social do seu cotidiano em benefícios acessíveis para a comunidade ao qual é inserido, tendo em vista proveitos coletivos que influenciarão diretamente na qualidade assistencial futura (Cunha et al., 2021). 
Portanto, vale ressaltar a importância da atenção primária e dos serviços ofertados pelo SUS, no qual caracteriza como uma forma mediadora, em uma simples roda de conversa, pode se identificar fatores que podem ser achados de uma doença, seja ela grave ou não.

Promover educação em saúde na atenção primária é muito importante, pois é um momento na qual podemos relatar algumas experiências vividas, e isso pode se tornar oportuno para que aquela mulher que está escutando, sinta-se motivada de realizar o " toque" para que ela mesma possa notar se há alguma diferença ou não no seu corpo.

Em síntese, a explanação do assunto permitiu que as mulheres pudessem desenvolver sua autonomia, sendo capaz de conhecer e opinar quanto às decisões para cuidar de si.

Explanar esses tópicos na atenção básica, contribuir para menores casos de mortalidade quanto ao câncer, falar abertamente de maneira natural torna a mulher acometida pela doença mais empoderada e forte para vencer a doença, ressaltando sempre que há cura, visto que na maioria das vezes por falta de apoio emocional, a mulher acaba achando que o diagnóstico de CA de mama será o fim de tudo.

Deste modo, as informações de práticas de autocuidado contribuem para a efetivação do processo de educação em saúde, possibilitando o incentivo de hábitos de vida mais saudáveis, desestimulando aos poucos hábitos inadequados e contemplando a necessidade de cada pessoa (Costa et al., 2020).

\section{Considerações Finais}

Com base no exposto, torna-se importante a abordagem deste tema na atenção primária, visto que é porta de entrada para muitas pessoas e detecção de doenças.

A importância de ações educativas voltadas para conscientização é de suma importância, a fim de rastrear casos de câncer de mama, podendo haver menos incidências dos casos de mortalidades causadas pelo câncer.

É fundamental alunos de diferentes cursos promoverem educação em saúde abordando temas relevantes na atenção primária de saúde, podendo contribuir como ferramenta de detecção precoce, a fim de conscientizar as mulheres quanto ao câncer de mama, e procurar ajuda ao serviço médico.

Há também vínculo profissional e paciente, no qual nesse momento de uma roda de conversa a fim de conscientizar, poder repassar informações que na maioria das vezes não são cessadas, com intuito de proporcionar a curiosidade dessas mulheres para que se toquem, e se notarem algo de errado, procurarem um serviço de saúde.

Por esse motivo, o referido estudo teve como objetivo descrever um relato de experiência sobre a educação em saúde sobre o câncer de mama para uma população carente de informações, pois acredita-se que a educação em saúde viabiliza possíveis reflexões e intervenções de expansão de conhecimento, bem como a necessidade da capacitação de profissionais para essas atividades.

Ademais, vale destacar a participação de acadêmicos dentro da comunidade, promovendo ações em saúde para lidar diretamente com diferentes situações, sendo essencial para o desenvolvimento de habilidades e competências necessárias para a profissão.

Em síntese, espera-se que este trabalho possa contribuir para futuras produções científicas que abordem a temática de prevenção ao câncer de mama, uma vez que essas ações são indispensáveis para promover o tratamento precoce, assim como a criação de estratégias que promovam a qualidade de vida da mulher. 
Research, Society and Development, v. 10, n. 14, e233101422137, 2021

(CC BY 4.0) | ISSN 2525-3409 | DOI: http://dx.doi.org/10.33448/rsd-v10i14.22137

\section{Referências}

Bernardes, N. B, Sá, A. C. F., Facioli, L. d. S., Ferreira, M. L., Sá, O. R., \& Costa, R. M. (2020). Câncer de Mama X Diagnóstico. $1-9$.

Bravo, B. S., Lopes, A. B. B., Tijolin, M. B., Nunes, P. L. P., Lenhani, T., Junior, S. F. D., \& Ceranto, D. d. C. F. B. (2021). Câncer de mama: uma revisão de literatura. 1-11

Câncer, I. N. d. (2021). Câncer de mama. 1-5.

Costa, P. V. D. P., Silva, J. M. L., Silva, L. C., Rocha, S. G., Costa, L. F., Soares, M. K. M., Santos, S. R. P., Lima, P. C., Rodrigues, B. N. B., Almeida, M. S.,Soeiro, J. S., Martins, L. Santos, G. L., A. C. S., \& Tavares, I. I. S. (2020). A educação em saúde como ferramenta no combate ao câncer de mama: relato de experiência. Research, Society and Development, 9(10).

Cunha, A. G., Silva, A. F. L. d., Mendes, A. P. d. S., Oliveira, A. K. C. d., Braga, B. S. C., Silva, B. C. M. d., Portilho, D. C., Portilho, D. C., Nascimento, J. L. M., Silva, J. M. L. d., Soeiro, J. d. S., Ferreira, L. F., Carvalho, M. A., Ferreira, P. d. S., Carvalho, P. H. C. d., \& Silva, R. R. d. (2021). Uso de metodologias ativas na promoção do autocuidado e adesão terapêutica com usuários de um centro de atenção psicossocial. Research, Society and Development, 10(1), 1-7.

Instituto Nacional de Câncer José Alencar Gomes da Silva. Ministério da Saúde (2021). Controle do câncer de mama. 1 - 19.

Leite, G. C., Ruhnke, B. F., \& Valejo, F. A. M. (2021). Correlação entre tempo de diagnóstico, tratamento e sobrevida em pacientes com câncer de mama: uma revisão de literatura. 1-5.

Lourenço, C. d. S., Silva, L. C. P., Laviola, G. M., Salles, D., Lopes, J. d. L., Waitzberg, A. F. L., Neto, R. A., \& Malinverni, A. C. d. M. (2020). Entendendo o câncer de mama: educação em saúde. 1-6.

Nascimento, J. L. d., Sousa, M. N. A. d., \& Alencar, T. P. (2020). Escalas sobre qualidade de vida em pacientes com câncer de mama. 1-20.

Santana, M. C., Rippel, J. R. d. S., \& Fortes, R. C. (2021). Boletim informativo para prevenção e diagnóstico precoce do câncer de mama. 1-9.

Silva, A. G., Medeiros, B. M., Rocha, B. M., Moro, C. C., Zingano, C. P., Bellan, L. M., \& Damin, A. P. S. (2021). Rastreamento do câncer de mama. 1-16.

Silva, P. A. d., \& Riul, S. d. S. (2015). Câncer de mama: fatores de risco e detecção precoce. 1-6.

Silva, R. R. D., Santos, T. S., Ramos, W. T., Barreiro, M. d. S. C., Mendes, R. B., \& Freitas, C. K. A. C. (2021). Ações do enfermeiro para prevenção e detecção precoce do câncer de mama. 1-5.

Teixeira, L. A., \& Neto, L. A. A. (2020). Câncer de mama no Brasil: medicina e saúde pública no século XX. 1-12.

Vieira, F. S., Portela, N. L. C., Sousa, G. C., Costa, E. S., Oliveira, D. E. P., \& Neiva, M. J. L. M. (2017). Inter-relação das ações de educação em saúde no contexto da Estratégia Saúde da Família: percepções do enfermeiro. Rev Fund Care Online, 9(4):1139-44. 